\title{
COVID-19 Hysteria: a "Chinese whispers" kind of game or a cause for psychiatric pathology?
}

\author{
Florin Mitu, Andreea Colessa, Iuliana Boboc, \\ Mădălina Niță, Maria Magdalena Leon Constantin
}

Florin Mitu - Professor, MD, PhD, Discipline of medical semiology, „Grigore T. Popa” University of Medicine and Pharmacy Iași, Rehabilitation Hospital Iași

Andreea Colessa - MD, cardiology resident, Cardiovascular Diseases Institute "George I.M. Georgescu" Iași

Iuliana Boboc - MD, oncology resident, "Sf. Dimitrie" Hospital Târgu Neamt,

Mãdãlina Niţă - MD, rheumatology resident, Rehabilitation Hospital Iași

Maria Magdalena Leon Constantin - Associate Professor, MD, PhD, Discipline of medical semiology, „Grigore T. Popa” University of Medicine and Pharmacy Iași, Rehabilitation Hospital Iași

\begin{abstract}
The media is unofficially considered the fourth largest power in the state. The motivation for this statement is remarkable. Through the press and audiovisual, the culture, perceptions, values, tendencies, decisions, and reactions of individuals are significantly influenced. But does it also affect psychiatric pathology? Although the sources of the news are required to be reliable, we do not always enjoy truthfulness or transparency. In the current social climate, unhappily animated by the hysteria of COVID-19, more and more people fall prey to anxiety, stress, depression, or even suicide. Fear of illness, social isolation, job loss, uncertainty looming over the near or distant future only complete this landscape of collective suffering. Fear arises from questions whose answer either does not exist or does not satisfy and can give rise to insecurity, despair, and sometimes even cruelty. Unfortunately, this whole collage of unhappiness is distinguished by a rapid increase. The economic crisis is expected to be heavier than in 2008, the unemployment rate has already reached alarming levels and continues to rise. This upheaval will add up in the future, as the lessons of past epidemics and pandemics teach us, to multiple pathologies of psychiatric origin. Post-traumatic stress syndrome, depression, anxiety, obsessive disorder and hypochondriac syndromes are just a few pieces of this mosaic.
\end{abstract}

\section{KEYWORDS:}

Mental health, anxiety, depression, mass-media, quarantine.. 


\section{INTRODUCTION}

Hysteria is, as Gisèle Harrus Révidi put it, "an evil which has passed through all ages, but which has nevertheless been adaptable to every culture" (1). Fear, in turn, has gained over time two major roles: that of protector but also that of the executioner: sometimes it saves from physical or mental injuries, other times it can cause irreversible damage to others or even to oneself.

December 2019 was the first time scientists discovered the new coronavirus (SARSCOV2). This microbiological entity would later be associated with multiple cases of pneumonia initially in the Wuhan region, China, and eventually, become a major global health problem. Soon researchers began to identify characteristics related to the virus, such as the mode of transmission, death rates, the origin of the virus. In parallel, public health representatives have begun to provide critical information on the spread of this new coronavirus so that, at both governmental and individual levels, measures can be taken to limit the spread of this virus (2).

The pandemic spotlights fall on us and reveal the naked human characters. During this time of trial for mankind, we have noticed that the planetary "cake" tends to be divided into three slices: one concentrates the wormwood essence of selfishness, evil, and despair, the second has gathered the sweetness of beauty, altruism, and self-sacrifice. And the third retained the tastelessness of indifference.

The abundance of aggressive news presented tragically, fake news, social isolation, the constant fear of illness and fatality, the uncertainty of a positive end, job loss, lack of money make up a color palette today with so many shades of gray that the whole picture of society fades and becomes dull.

A new threat has emerged among the population, the psychological suffering resulting from repeated exposure to information received from the media. This has implications not only for the immediate suffering of the population that is already socially and economically affected but it also has effects on physical and mental health over time (2).

Prospective studies have shown that exposure to stress during and after a crisis is associated with a greater number of physical and mental side effects over time (2).

Affecting public health can lead to imbalances both at the individual level (leading to, for example, insecurity, confusion), and at the community level (especially due to economic losses, job closures, and schools). These effects can be translated into an increase in the incidence of emotional reactions, unhealthy habits such as substance abuse and non-compliance with the measures applied to the population that has already contracted the disease, but with the measures for general population (3).

\section{IN LOVE WITH THE MEDIA? MAYBE IT'S TOXIC LOVE}

During a period of crisis, the population depends on the information received from the media and it is imperative that it be a safe and available source to provide risk assessments and recommendations. The population tends to form accurate perceptions of risk when the facts are known and communicated to the public in an effective way through the media. In the absence of public information, ambiguity may exacerbate insecurity among the population (2). This has been observed in the context of the AH1N1 virus crisis, with uncertainty and lack of public information leading to increased anxiety (4). When ambiguity is combined with an invisible enemy such as a virus, fear and anxiety may be exacerbated.

The paradox is that although health professionals and journalists are working to assess risks, recommendations, and morbidity and mortality in order to get the most complete picture of the global critical situation, a new threat can be seen and is amplified day after day: the psychological suffering caused by repeated exposure to the media. 
After critical events most people are optimistic and do not give into psychosocial stress, however a part of the population will develop the so-called post-traumatic stress disorder (PTSD). Medical conditions such as infection with this new coronavirus are not a current criterion for trauma to be included in this syndrome, but it can cause mental disorders such as depression and anxiety (3). In a recent US study of the terrorist attacks of 11 September 2001, increased hours of exposure to information received through television in the days following the events of that date were associated with increased posttraumatic stress and onset mental illness 2-3 years later (2).

Another example would be the episode of the Boston bombings, which showed a strong association between the amount of information received from the media and the appearance of symptoms of acute stress. The anxiety rate is 3 times higher in those exposed to media than those who had gone through those events. In addition to the amount of information received from the media, its content is also important. Exposure to bloody images showed an increase in post-traumatic stress and anxiety for 6 months after the events in Boston (2).

\section{FAKE NEWS, BUT REAL PATHOLOGIES}

During a crisis, people often look for information about the event to stay up to date with everything that happens. However, when information on official channels is missing or disseminated in a disorganized manner, people may be exposed to misleading information on social media (3).

A recent study of the Chinese population, which included 4872 participants from 31 autonomous regions, all over the age of 18 , showed that more than $80 \%$ of participants reported frequent exposure to information received from social media during coronavirus pandemic. $48.3 \%$ of participants had episodes of depression, $22.6 \%$ reported anxiety and $19.4 \%$ reported both depression and anxiety. Depression was assessed through the Chinese version of the WHO-5 - Five
Well Being Index, and consisted of evaluating five positive elements that reflect the presence or absence of well-being of individuals, rather than the presence of symptoms related to depression. A total score of 13 indicated depression. It was observed that individuals aged between 21-40 years were the most affected compared to those whose age was less than 20 years (5).

Regarding anxiety, it was assessed according to the Chinese version of the generalized anxiety disorder scale (GAD -7). The highest rate of anxiety was observed in the 31-40 years age category. Depression and anxiety were reported in $19.4 \%$ of the surveyed population, higher values being observed in the $31-40$ years age category (5).

This demonstrates a strong association between the increased prevalence of mental health problems and frequent exposure to social media (EMS). As media reports can lead to emotional disturbances, contact with pandemic news should be limited (2).

In turn, the public should be advised to avoid speculative stories and limit repeated exposure to media stories that provide little new information. Social media platforms must also monitor and eliminate any false information, promoting credible sources such as the WHO or the CDC (2). In Italy, $4.8 \%$ of the total news and online posts related to coronavirus were false or inaccurate. Percentage value was calculated based on the average volume of posts and articles published by the media, including social media posts. The peak in false news reporting was recorded in the early stages of the pandemic at the end of January 2020, accounting for $7.3 \%$ of coronavirus information (6). In this context, on April 7, 2020, the social networking platform WhatsApp took the decision to restrict the number of people to whom it can be shared, in order to limit misinformation (7).

\section{WORK HARD ... SUFFER HARD?}

Some groups of people may be more vulnerable than others to the psychosocial effects of a pandemic. This category includes 


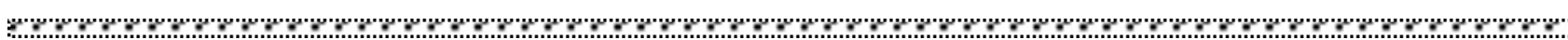

patients who have already contracted the disease, patients at high risk of developing the disease such as the elderly, those with a deficient immune system, but also patients with pre-existing mental illness (3).

Medical staff is also a vulnerable group, especially to emotional stress, given the risk of exposure to the virus, the concern about the risk of illness, the lack of medical equipment, long working hours.

Also, health workers can develop mental disorders in connection with the stressful events to which they are subjected. During 2003, during the SARS-Cov epidemic in Singapore, $27 \%$ of the medical staff developed mental symptoms (8). Similarly, during the 2014 Ebola epidemic in the Sierra and the Democratic Republic of the Congo in 2018, there were increased levels of anxiety among medical staff who came into contact with infected people (9).

In the case of the pandemic caused by COVID19, Wuhan medical staff faced a series of critical events related to the increased risk of infection, lack of protective equipment, frustration, discrimination, isolation, lack of contact with family members and exhaustion. This situation causes mental health problems such as anxiety, insomnia, depression, stress, anger and fear (1).

It has been observed that medical staff in emergency units are more likely to develop severe symptoms of PTSD even compared to staff in psychiatric wards. The prevalence of PTSD in the general population varies from $4 \%$ to $41 \%$, while the prevalence of depression increased by $7 \%$ after this epidemic. There are certain factors that increase the risk: gender - women, poor socioeconomic status, interpersonal conflicts, or frequent use of social media (1).

\section{SOCIAL ISOLATION TO REMAIN HEALTHY... BUT DEPRESSED?}

In the context of the COVID-19 pandemic, psychosocial assessment should include questions about stressors (exposure to sources of infection, infection of family members, loss of a loved one, physical distancing), side effects, and psychosocial effects. Such as psychosomatic concerns, insomnia, domestic violence (3).

Quarantine is the restriction of the movement of people or goods in order to limit the spread of a certain disease.

Five studies compared different psychological aspects in the case of quarantined and nonquarantined people (2). A study (13) of hospital staff who may have come in contact with SARS found that as soon as the quarantine period (9 days) ended, there was an increase in symptoms of acute stress disorder. In the same study, quarantined staff reported significantly more burnout, social detachment, anxiety in the presence of febrile patients, irritability, insomnia, impaired concentration and indecisiveness, issues that led to a decrease in work performance, reluctance to work or even deciding to resign. In another study, (4) the effect of quarantine was a predictor of post-traumatic stress disorder symptoms in hospital employees even 3 years later. About $34 \%$ of horse farm owners quarantined for a few weeks due to an outbreak of horse flu have reported a high level of psychological distress, compared to around $12 \%$ in the Australian general population (5).

A study (6) comparing the symptoms of posttraumatic stress in quarantined parents and children with those not-quarantined found that the average scores of post-traumatic stress were four times higher in children who were quarantined. $28 \%$ of quarantined parents in this study reported symptoms that justify the diagnosis of post-traumatic stress disorder, compared to $6 \%$ of parents who were not quarantined. Another study (7) that examined the presence of depressive symptoms in hospital staff 3 years after quarantine, found that $9 \%$ of the entire sample reported major depressive symptoms. In the group with high depressive symptoms, almost $60 \%$ were quarantined, but only $15 \%$ in the group with low depressive symptoms were quarantined. 
Two studies looked at the long-term effects of quarantine. Three years after the outbreak of SARS, the association of alcohol abuse or dependence on other harmful substances has been observed in health workers in high-risk areas (8).

A qualitative study (9) reported that several participants described long-term behavioral changes after the end of the quarantine period, such as frequent hand washing, avoidance of crowds. For some, a return to normalcy has been delayed for months.

The evaluation of the possible psychological sequelae of the quarantined persons revealed numerous results, as highlighted above. Possible stressors included longer restraint period, inadequate stockpile, financial losses. Some of these patients will need a full mental health assessment after this period, while others may benefit from supportive interventions designed to promote well-being (3).

For people with a vulnerable mental status, mental health care and monitoring should be provided even 6 months after the end of the isolation period. The assistance should include accurate information as well as appropriate consumables for the subjects, including clothing, food, and accommodation if needed. In light of the growing economic crisis and the general uncertainty caused by this epidemic, suicidal ideation may arise and requires rapid intervention by the specialist (10).

\section{HEATHY + NO FOOD = MENTAL HEALTH PROBLEMS}

Financial losses can be a significant problem during a pandemic.

In the United States, the unemployment rate rose from 3.5\% in February to 17\% in March, totaling more than 9 million people who lost their jobs, and this data needs to be updated, which will lead to an increase, because the statistics describe the situation only for the first two weeks of March (9).

More than half of low-income American households report that at least one family member has lost their job or their income has been reduced due to the COVID-19 pandemic. Only $23 \%$ of them state that they have an emergency fund that will last them for 3 months (8).

A study (22) of people in quarantine due to the potential Ebola contact found that although participants received financial assistance, some felt that the amount was insufficient and that it came too late. A study (9) of people quarantined in Toronto during SARS reported multiple financial difficulties because employers or the government compensated them, but reimbursement was slow and insufficient and they could not easily cope with the losses.

After the economic crisis of 2008, there was an increase in the suicide rate by $4.2 \%$ in European countries and $6.4 \%$ in 10 countries in America. In Europe, the most affected age group was 15-24 years old (with an increase of $11.7 \%$ ), and in the American countries, men aged 45-64 years old had the highest increase in the suicide rate by $5.2 \%$ (3).

A study conducted in Estonia found an increase in the prevalence of depression after the 2008 economic crisis from 0.77 in 2006 to 1.22 in 2008, with men in the 35-64 age group and women aged 50-64 being the most affected age ranges (10).

Participants with a low annual income had a significantly higher rate of post-traumatic stress and depressive disorders. This is probably due to the fact that they are more likely to be affected by a temporary financial loss than those with significantly higher incomes (11). 


\section{CONCLUSION}

It is said that "hard times" are those that solidify a relationship or destroy it. We have no doubt that a pandemic is a difficult time. More surprising, however, is the fact that "good" measures (informing the population through the media, quarantine, social isolation) taken to prevent the spread of the virus also bring "bad" results (the decline of mental status for some people). In the acute moment of the pandemic, we expect an increase in anxiety, abuse of toxic substances, insomnia, fear, concentration problems. But the end of the pandemic will not bring the end of problems. Increasing suicide rates, depression, and PTSD are just a few of the issues we will have to deal with for at least 2-3 years.

In the end, all we have left is the hope of learning something about empathy, optimism, courage, and self-sacrifice from the harsh lesson the pandemic was, and perhaps we aquire the idealism that united we are stronger.

\section{ACKNOWLEDGEMENTS AND DISCLOSURES}

The authors state that they are no declared conflicts of interest regarding this paper.

\section{REFERENCES}

1. Garfin D. R., Silver R. C., Holman E. A.: The novel coronavirus (COVID-2019) outbreak: Amplification of public health consequences by media exposure. Health Psychology 2020; 39(5): 355-357.

2. Pfefferbaum B., Schonfeld D., Flynn B.W., et al.: The H1N1 crisis: a case study of the integration of mental and behavioral health in public health crisis. Disaster Med Public Health Prep 2012; 6:67-71.

3. https://www.statista.com/statistics/1109490/share-of-coronavirus-fake-news-italy/

4. https://www.theverge.com/2020/4/7/21211371/whatsapp-message-forwarding-limits-misinformation-coronavirusindia.

5. Lee S.M., Kang W.S., Cho A.-R., Kim T., Park J.K.: Psychological impact of the 2015 MERS outbreak on hospital workers abd quarantined hemodialysis patients. Comprehensive Psychiatry 2018, 87: 123-127.

6. Park J.-S., Lee E.-H., Park N.-R., Choi Y.H.: Mental health of nurses working at a government-designated hospital during a MERS-CoV outbreak: A cross- sectional study. Archives of Psychiatric Nursing 2018, 32:2-6.

7. Kang L., Li Y., Hu. S., Chen M., Yang C., Yang B.: The mental health of medical workers in Wuhan, China dealing with the 2019 novel coronavirus. The Lancet Psychiatry 2020, 7: E14.

8. https://www.theguardian.com/business/2020/apr/02/us-unemployment-coronavirus-economy.

9. https://www.pewsocialtrends.org/2020/04/21/about-half-of-lower-income-americans-report-household-job-orwage-loss-due-to-covid-19/.

10. Chang S.-S., Stuckler D., Yip P., Gunnell D.: Impact of 2008 global economic crisis on suicide: time trend study in 54 countries BMJ 2013; 347:f5239.

11. Leinsalu M, Reile R, Stickley A.: Economic fluctuations and long-term trends in depression: a repeated crosssectional study in Estonia 2004-2016. J Epidemiol Community Health. 2019;73(11):1026-1032.

\section{Correspondence}

Maria Magdalena Leon Constantin,

Associate Professor, MD, PhD, Discipline of medical semiology, „Grigore T. Popa” University of Medicine and Pharmacy Iași, ROMANIA; Rehabilitation Hospital Iași, leon_mariamagdalena@yahoo.com 